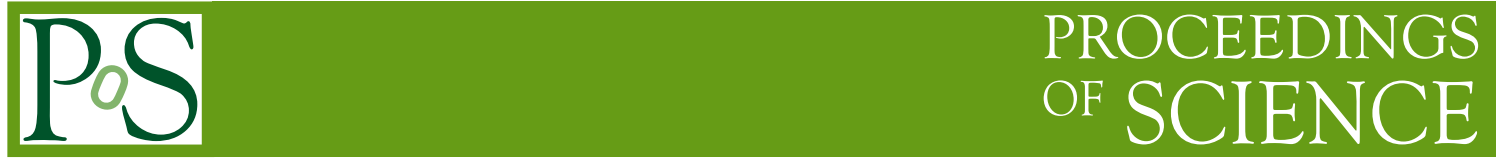

\title{
Search for new signals in lepton pairs and photon pairs with the ATLAS detector
}

\section{Xabier Anduaga*}

On behalf of the ATLAS Collaboration

Instituto de Física La Plata, Universidad Nacional de La Plata and CONICET

E-mail: xabier.anduaga@cern.ch

\begin{abstract}
A brief overview of searches for high mass exotic signals using proton-proton collision data from the LHC collected by the ATLAS experiment during 2011 is presented. Several final states are considered including a pair of leptons or photons and a lepton with missing transverse energy. No evidence of new signals was found and exclusion limits were set in the context of several new physics models.
\end{abstract}

36th International Conference on High Energy Physics,

July 4-11, 2012

Melbourne, Australia

\footnotetext{
${ }^{*}$ Speaker.
} 


\section{Introduction}

The Standard Model (SM) of particle physics has been describing remarkably well the data obtained from high energy experiments. Nonetheless, arguments based on unitarity require new physics at the $\mathrm{TeV}$ scale, which could be accessed with $7 \mathrm{TeV}$ center-of-mass energy proton-proton (pp) collisions from the Large Hadron Collider (LHC). New signals decaying into a pair of photons or charged leptons (electrons and muons), or into a charge lepton and a neutrino are of particular interest due to the clean experimental signature, excellent mass resolution and modest backgrounds. In this proceedings an overview of the search for such signals performed at the ATLAS experiment [1] with pp collision data collected during 2011 is presented.

\section{Search for heavy dilepton signatures}

Several extensions of the SM predict heavy resonances decaying to a charged lepton-anti lepton pair. These include neutral gauge bosons $Z^{\prime}$ [2, 3, 4], techni-mesons [5, 6] and Kaluza-Klein (KK) graviton excitations in the Randall-Sundrum (RS) Model [7]. A search for a Sequential Standard Model (SSM) $Z^{\prime}$ and a RS graviton was carried out using around $5 \mathrm{fb}^{-1}$ of data both in the dielectron and dimuon channels [8]. The invariant mass distribution for each channel is shown in Figure 1, where only events containing a pair of well isolated electrons or muons passing a full set of selection cuts are included. The main background from SM, containing $Z / \gamma^{*}$ Drell-Yan, diboson, $t \bar{t}$ and multijet production is also included. The background predictions were obtained at next-to-next-to-leading order (NNLO) for $Z / \gamma^{*}$ and dibosons, at approximately NNLO for $t \bar{t}$, and from data for multijet production. The data were found to be in very good agreement with SM expectations and limits were set on both models. For the $Z^{\prime}$ models, the $95 \%$ confidence level (CL) limit on the cross-section times branching ratio $(\sigma B)$ as a function of the mass is shown in Figure 2. The observed limits and the expected limits with the corresponding $\pm 1 \sigma$ and $\pm 2 \sigma$ bands are included, together with the theoretical predictions for some benchmark models. For the SSM $Z^{\prime}$, masses up to $2.22 \mathrm{TeV}$ are excluded, while for the E6 family of models [2] the exclusion region goes up between 1.79 to $1.97 \mathrm{TeV}$ depending on the mixing angle. RS gravitons with a $k / \bar{M}_{P l}$ dimensionless coupling [7] value of $0.01(0.1)$ are also excluded up to a mass of $0.92(2.16)$ $\mathrm{TeV}$.

A similar previous study was performed with a subsample of the 2011 data corresponding to roughly $1.1 \mathrm{fb}^{-1}$ and the results interpreted in the context of the techni-meson and quark compositeness models $[9,10,11]$. For the techni-mesons [12], the excluded area in the $m\left(\rho_{T} / \omega_{T}\right)-m\left(\pi_{T}\right)$ plane is shown on Figure 3. For a splitting in techni-meson masses of $100 \mathrm{GeV}$, the $95 \% \mathrm{CL}$ lower limit on $m\left(\rho_{T} / \omega_{T}\right)$ is found to be $470 \mathrm{GeV}$. Compositeness models can be described by a contact interaction and would appear as a non-resonant excess in the dilepton invariant mass distribution. In this case, the compositeness scale $\Lambda$ is excluded up to values of 10.2 (8.8) $\mathrm{TeV}$ for destructive (constructive) interference hypothesis when using a flat prior of $1 / \Lambda^{2}$ [13].

\section{Search for heavy diphoton signatures}

A search for new physics in the diphoton mass distribution $\left(m_{\gamma \gamma}\right)$ was carried out using $4.9 \mathrm{fb}^{-1}$ of data [16]. Events were selected requiring at least two well isolated photons with a mass of the 

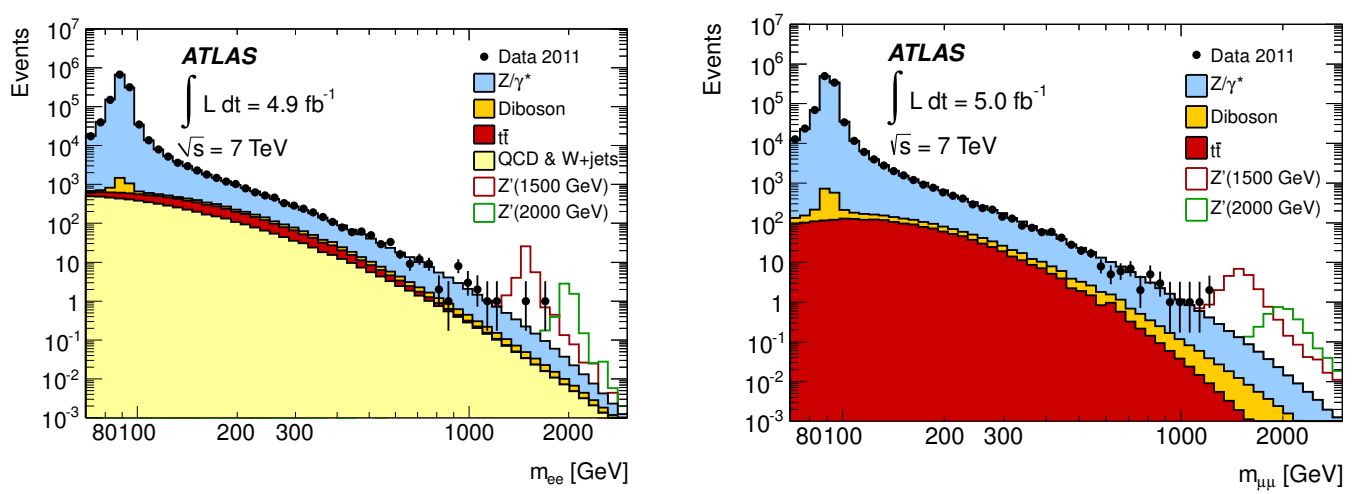

Figure 1: Dielectron (left) and dimuon (right) invariant mass $\left(m_{l l}\right)$ distributions after final selection, compared with the stacked sum of all expected backgrounds, with two example $Z_{S S M}^{\prime}$ signals overlaid [8]. The bin width is constant in $\log \left(m_{l l}\right)$.
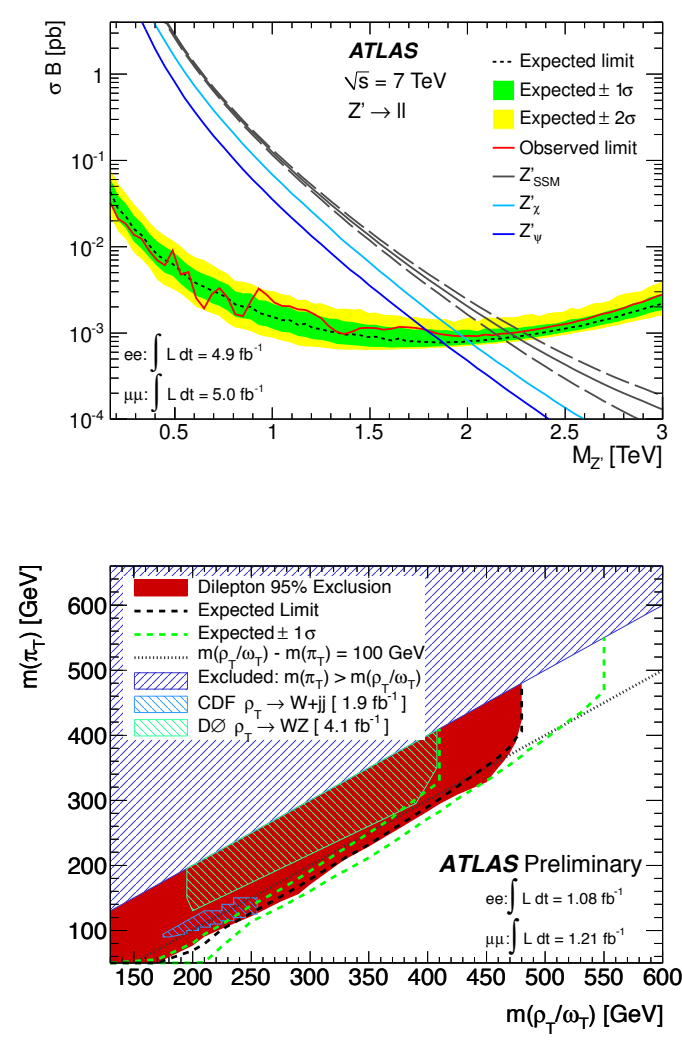

Figure 2: Observed and expected 95\% CL limits on $\sigma B$ and expected $\sigma B$ for $Z_{S S M}^{\prime}$ production and the two E6-motivated $Z^{\prime}$ models with lowest and highest $\sigma B$ for the combination of the dielectron and dimuon channels [8]. The dashed lines around the $Z_{S S M}^{\prime}$ predictions represent the theoretical uncertainty, which is similar for the other theory curves.

Figure 3: The 95\% CL excluded region as a function of the assumed $\pi_{T}$ and $\rho_{T} / \omega_{T}$ masses is shown in red [12]. The dotted line corresponds to $m\left(\rho_{T} / \omega_{T}\right)-m\left(\pi_{T}\right)=100 \mathrm{GeV}$. The dashed line shows the expected limit with the green dashed lines showing the $\pm 1 \sigma$ bands. The hashed region where $m\left(\pi_{T}\right)>m\left(\rho_{T} / \omega_{T}\right)$ is excluded by theory. Also shown are recent results from CDF [14] and DO [15].

pair larger than $142 \mathrm{GeV}$. The observed $m_{\gamma \gamma}$ spectrum can be seen in Figure 4 together with the expected SM background. No significant excess of events is observed neither in a resonant or nonresonant final state. This result allows to set constraints on RS graviton production and on virtual graviton exchange processes as proposed by Arkani-Hamed, Dimopoulos and Dvali (ADD) [17]. RS gravitons with masses up to 1.0 (2.06) $\mathrm{TeV}$ are excluded at $95 \% \mathrm{CL}$ for a $k / \bar{M}_{P l}$ coupling value of $0.01(0.1)$. In the context of the ADD model, a 95\% CL limit on the number of events above a mass threshold of $1217 \mathrm{GeV}$ was set and then translated into a limit on the fundamental Planck scale $\left(M_{S}\right)$. The results are represented in Figure 5. Lower limits of between $2.52 \mathrm{TeV}$ 


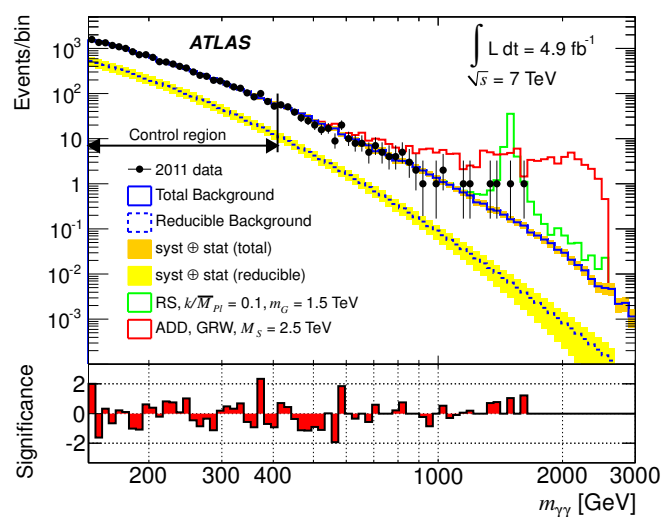

Figure 4: Observed invariant mass distribution of diphoton events [16]. Superimposed are the SM background expectation and the expected signals for two examples of RS and ADD models. In addition to the total background, the contribution from the reducible component is shown. The black arrow indicates the boundaries of the control region. To compensate the rapid decrease of the spectrum, the bins have been chosen to have constant logarithmic width. The bin-by-bin significance of the difference between data and predicted background is shown below the main plot. Following the convention of Eur. Phys. J. Plus 127 (2012) 25 , the significance is set to zero for bins with insignificant deviations with respect to a small expected background.

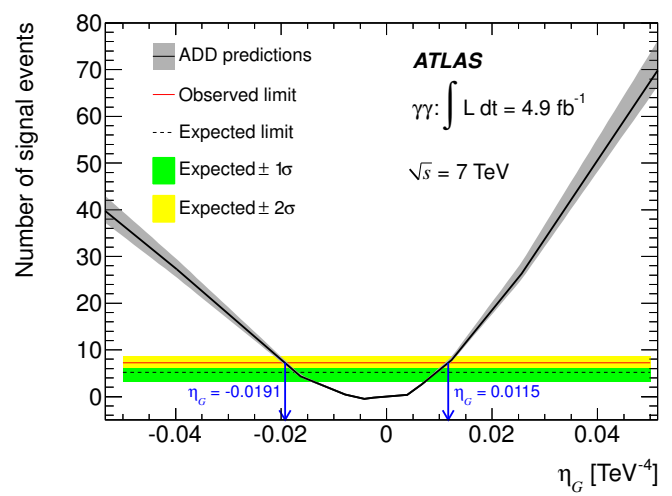

Figure 5: Number of signal events as a function of $\eta_{G}$ [16]. The parameter $\eta_{G}$ measures the strength of gravity in the presence of extra dimensions and it is proportional to $M_{s}^{-4}$, where the exact value depends on the theoretical formalism considered. The solid horizontal line corresponds to the observed limit, the dashed line corresponds to the expected limit and the bands to the $\pm 1 \sigma$ and $\pm 2 \sigma$ uncertainty on the expected limit. The black curve corresponds to Monte Carlo predictions, while the band around it illustrates the theoretical uncertainties. When the prediction is greater than the limit line, the corresponding value for $\eta_{G}$ is excluded.

and $3.92 \mathrm{TeV}$ are set on $\mathrm{M}_{S}$, depending on the number of extra dimensions and the theoretical formalism used $[18,19,20]$.

\section{Search for a heavy gauge boson decaying into a lepton and a neutrino}

In this study [21] a search for evidence of a SSM heavy charged gauge boson $\left(W^{\prime}\right)[22,23,24]$ is performed by analyzing samples of events with exactly one isolated electron or muon together with large missing transverse momentum $\left(E_{T}^{\text {miss }}\right)$. A total of $4.7 \mathrm{fb}^{-1}$ of data was used. The observed transverse mass distribution, defined as $m_{T}=\sqrt{2 p_{T} E_{T}^{m i s s}\left(1-\cos \phi_{l v}\right)}$, where $\phi_{l v}$ is the azimutal angle between the lepton momentum and $E_{t}^{\text {miss }}$ vectors, is shown in Figure 6 for each of the channels. The data were found to be compatible with the SM expectation. The 95\% CL limit on $\sigma B$ as a function of the mass is shown in Figure 7. The combination of channels allows to exclude 
the SSM $W^{\prime}$ for masses below $2.55 \mathrm{TeV}$, while excited chiral bosons $\left(W^{*}\right)$ with equivalent coupling strength are excluded for masses up to $2.42 \mathrm{TeV}$.
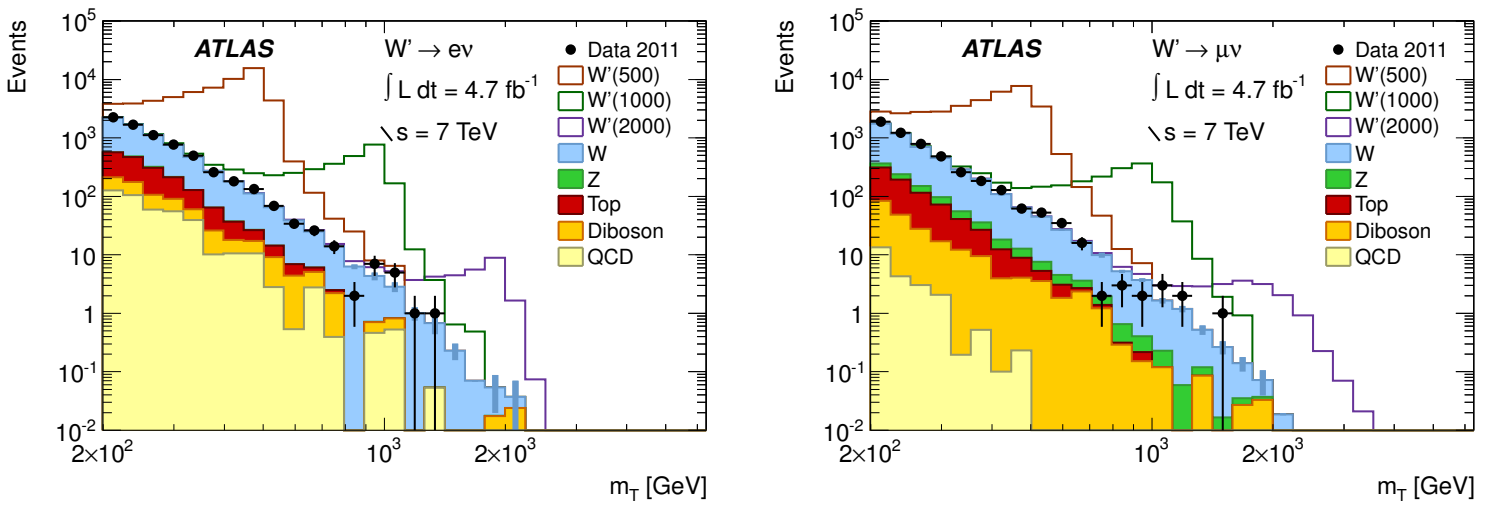

Figure 6: Spectra of $m_{T}$ for (left) the electron channel and (right) the muon channel for events with $m_{T}>$ $200 \mathrm{GeV}$ after event selection [21]. The points represent the data and the filled histograms show the stacked backgrounds. Open histograms are $W^{\prime} \rightarrow l v$ signals added to the background with masses in $\mathrm{GeV}$ indicated in parentheses in the legend. The multijet background estimated from data is also shown. The signal and other background samples are normalized using the integrated luminosity of the data and the NNLO cross sections.

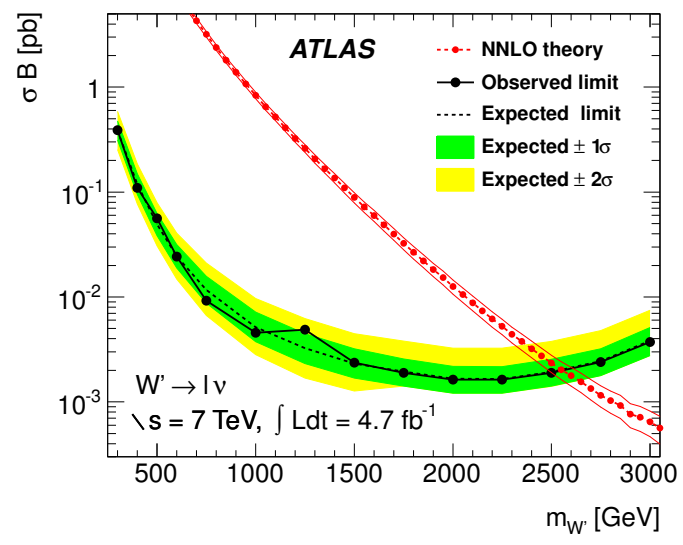

Figure 7: Observed and expected limits on $\sigma B$, for $W^{\prime} \rightarrow l v$ in the combination of electron and muon channels assuming the same branching fraction for both [21]. The predicted value for $\sigma B$ (NNLO) and its uncertainty is also shown.

\section{References}

[1] ATLAS Collaboration, JINST 3, S08003 (2008).

[2] D. London and J. L. Rosner Phys. Rev. D34 (1986) 1530.

[3] P. Langacker Rev. Mod. Phys. 81 (2009) 1199-1228.

[4] J. Erler, P. Langacker, S. Munir, and E. R. Pena JHEP 0908 (2009) 017.

[5] K. D. Lane and S. Mrenna Phys. Rev. D 67 (2003) 115011. 
[6] E. Eichten and K. Lane Phys. Lett. B 669 (2008) 235-238.

[7] L. Randall and R. Sundrum Phys. Rev. Lett. 83 (1999) 3370-3373.

[8] ATLAS Collaboration, accepted by JHEP, arXiv:1209.2535 [hep-ex].

[9] E. Fermi, Nuovo Cim. 11, 1 (1934); Z. Phys. 88 (1934) 161.

[10] E. Eitchen, K. Lane, and M. Peskin, Phys. Rev. Lett. 50 (1983) 811.

[11] J. Beringer et al. (Particle Data Group), Phys. Rev. D86 (2012) 010001.

[12] ATLAS Collaboration, ATLAS-CONF-2011-125, https://cdsweb.cern.ch/record/1383787.

[13] ATLAS Collaboration, Phys. Lett. B 712 (2012) 40.

[14] CDF Collaboration, T. Altonen et al., Phys. Rev. Lett. 104 (2010) 111802.

[15] D0 Collaboration, V. Abazov et al., Phys. Rev. Lett. 104 (2010) 061801.

[16] ATLAS Collaboration, submitted to Phys. Lett. B, arXiv:1210.8389 [hep-ex].

[17] N. Arkani-Hamed, S. Dimopoulos, and G. Dvali, Phys. Lett. B 429 (1998) 263-272.

[18] G. F. Giudice, R. Rattazzi, and J. D. Wells, Nucl. Phys. B 544 (1999) 3-38.

[19] T. Han, J. D. Lykken, and R.-J. Zhang, Phys. Rev. D 59 (1999) 105006.

[20] J. L. Hewett, Phys. Rev. Lett. 82 (1999) 4765-4768.

[21] ATLAS Collaboration, submitted to Eur. Phys. J. C, arXiv:1209.4446 [hep-ex].

[22] G. Altarelli, B. Mele, and M. Ruiz-Altaba Z.Phys. C45 (1989) 109-121.

[23] M.V. Chizhov, V.A. Bednyakov, J.A. Budagov Phys. At. Nucl. 71(2008) 2096-2100.

[24] M. Chizhov, G. Dvali Phys. Lett. B 703 (2011) 593-598. 